\title{
Smart Contract Vulnerability Detection Using Graph Neural Networks
}

\author{
Yuan Zhuang ${ }^{1, *}$, Zhenguang Liu ${ }^{1, *}$, Peng Qian ${ }^{1, *}$, Qi Liu ${ }^{2}$, Xiang Wang ${ }^{3}$, Qinming He ${ }^{4}$ \\ ${ }^{1}$ Zhejiang Gongshang University \\ ${ }^{2}$ University of Oxford \\ ${ }^{3}$ National University of Singapore \\ ${ }^{4}$ Zhejiang University \\ zhuangyuan2020@outlook.com, liuzhenguang2008@gmail.com, messi.qp711@gmail.com, \\ qi.liu@cs.ox.ac.uk, xiangwang1223@gmail.com, hqm@zju.edu.cn
}

\begin{abstract}
The security problems of smart contracts have drawn extensive attention due to the enormous financial losses caused by vulnerabilities. Existing methods on smart contract vulnerability detection heavily rely on fixed expert rules, leading to low detection accuracy. In this paper, we explore using graph neural networks (GNNs) for smart contract vulnerability detection. Particularly, we construct a contract graph to represent both syntactic and semantic structures of a smart contract function. To highlight the major nodes, we design an elimination phase to normalize the graph. Then, we propose a degree-free graph convolutional neural network (DR-GCN) and a novel temporal message propagation network (TMP) to learn from the normalized graphs for vulnerability detection. Extensive experiments show that our proposed approach significantly outperforms state-of-the-art methods in detecting three different types of vulnerabilities.
\end{abstract}

\section{Introduction}

Blockchain technology is developing rapidly due to its decentralization and tamper-free nature [Tsankov et al., 2018]. A blockchain is essentially a distributed and shared transaction ledger, maintained by all the miners in the blockchain network following a consensus protocol [Sankar et al., 2017]. Smart contracts are programs automatically running on the blockchain. However, ill-designed smart contracts expose vulnerabilities, which are perfect targets for network attacks. One notable example is the DAO event, where the hackers exploit the reentrancy bug of The DAO contract to steal 3.6 million Ether (Cryptocurrency of Ethereum). The case is not isolated and several security vulnerabilities are discovered and exploited every few months ${ }^{\dagger}$. According to the statistics of SlowMist Hacked ${ }^{\ddagger}$, blockchain networks have suffered more

\footnotetext{
${ }^{*}$ The first three authors are of equal contribution to this work. Zhenguang Liu contributes to the idea, Yuan Zhuang and Peng Qian contribute to implements and datasets. Zhenguang Liu is the corresponding author.

${ }^{\dagger}$ The dao website, 2016. http://etherscan.io/address/0xbb9bc244 d798123fde783fcc1c72d3bb8c189413

${ }^{\ddagger}$ Slowmist hacked website, 2019. https://hacked.slowmist.io/en/.
}

than 10 billion USD losses due to the security issues of smart contracts.

Current approaches for smart contract vulnerability detection are mainly inspired by existing testing methods from the programming language community, revolving around symbolic execution [Luu et al., 2016; Tsankov et al., 2018] and dynamic execution methods [Jiang et al., 2018; Liu et al., 2018b]. We scrutinized the released implementation of existing methods, and empirically observe that they suffer from two key problems. First, existing methods heavily rely on several expert-defined hard rules (or patterns) to detect smart contract vulnerability. However, expert rules are error-prone and some complex patterns are non-trivial to be covered. Crudely using several hard rules leads to high false-positive and false-negative rates, and crafty attackers may easily bypass the rules to perform attacks. Second, since the rules are contributed by a few 'centralized' experts who develop the detection tools, their scalability is inherently limited. As the number of smart contracts is increasing rapidly, it is impossible for a few experts to sift through all the contracts to design precise rules, while the knowledge of other 'decentralized' experts cannot be incorporated to improve the model.

Our method. To address these problems, we propose novel methods beyond the rule-based framework. Specifically, we characterize the source code of a smart contract as a contract graph according to the data- and control- dependencies between program statements. Nodes in the graph represent critical function invocations or variables while edges capture their temporal execution traces. Since most GNNs are inherently flat during information propagation, we design an elimination phase to normalize the graph. We extend GCN to a degree-free GCN (DR-GCN) to handle the normalized graphs. Further, we take into account the distinct roles and temporal relationships of different program elements and propose a novel temporal message propagation network (TMP). We conducted extensive experiments on more than 300,000 real-world smart contract functions, results show that our approaches significantly and consistently outperform state-ofthe-art methods on the detection of different types of vulnerabilities including reentrancy, timestamp dependence, and infinite loop vulnerabilities. Our implementations are released to facilitate future research. 


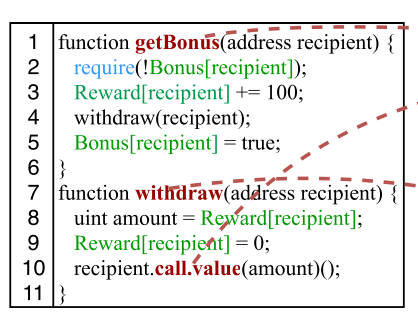

(a) Contract snippet

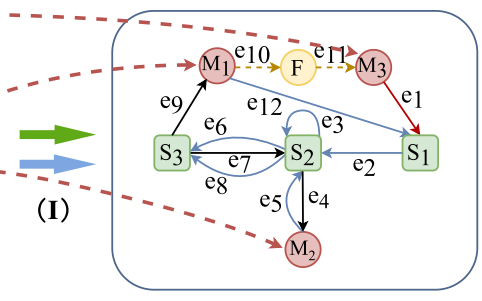

(b) Contract graph

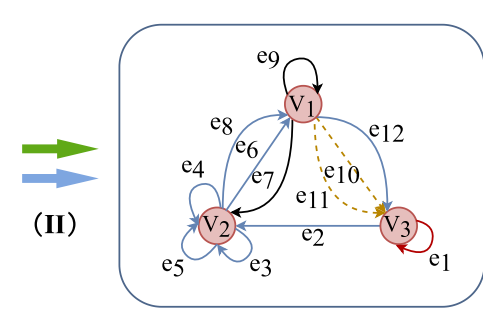

(c) Normalized graph

Temporal Edges

\begin{tabular}{|c|c|c|c|c|}
\hline & Vstart & Vend & Orde & r Type \\
\hline & $\mathrm{V}_{3}$ & $\mathrm{~V}_{3}$ & 1 & RG \\
\hline & $V_{3}$ & $\mathrm{~V}_{2}$ & 2 & $\mathrm{AC}$ \\
\hline & $\mathrm{V}_{2}$ & $\mathrm{~V}_{2}$ & 3 & FW \\
\hline & $V_{1}$ & $\dddot{\mathrm{V}_{3}}$ & 10 & FB \\
\hline & $\mathrm{V}_{1}$ & $\mathrm{~V}_{3}$ & 11 & FB \\
\hline & $\mathrm{V}_{1}$ & $\mathrm{~V}_{3}$ & 12 & $\mathrm{AG}$ \\
\hline \multicolumn{5}{|c|}{ Clustered Nodes } \\
\hline \multicolumn{2}{|c|}{ us $\mathrm{Maj}^{\mathrm{Sec}}$} & $S_{\text {in }}$ & \multicolumn{2}{|c|}{$S_{\text {out }}$} \\
\hline & $\mathrm{M}_{1}$ & \multirow{2}{*}{\multicolumn{2}{|c|}{$\begin{array}{l}\mathrm{S}_{2}, \mathrm{~S}_{3} \\
\mathrm{~S}_{1}, \mathrm{~S}_{2}\end{array}$}} & $\mathrm{~S}_{1}$ \\
\hline & $\mathrm{M}_{2}$ & & & $\mathrm{~S}_{2}$ \\
\hline & $\mathrm{M}_{3}$ & \multicolumn{3}{|c|}{$\mathrm{F}$} \\
\hline
\end{tabular}
Major Node $\square$ Secondary Node Fallback Node $\longrightarrow$ Forward Edge $\longrightarrow$ Control-flow Edge $\longrightarrow$ Data-flow Edge $-->$ Fallback Edge

Figure 1: The graph generation and normalization phases of our method. (a) shows the source code of a smart contract; (b) visualizes the graph extracted from the source code. Nodes in circle denote major nodes and nodes in square represents secondary nodes. (c) demonstrates the graph after normalization.

Contributions. To summarize, our key contributions are: i) We introduce a novel temporal message propagation network (TMP) and a degree-free GCN (DR-GCN) to automatically detect smart contract vulnerabilities. ii) We propose to characterize the contract function source code as contact graphs, and explicitly normalize the graph for highlighting the key nodes. iii) Our methods set the new state-of-the-art performance on smart contract vulnerability detection, and overall provide insights into the challenges and opportunities.

\section{Problem Statement}

Problem formulation. Presented with the source code of a smart contract, we are interested in developing a fully automated approach that can detect vulnerabilities at the function level. That is, we are to estimate the label $\hat{y}$ for each smart contract function $S C$, where $\hat{y}=1$ represents $S C$ has a vulnerability of a certain type while $\hat{y}=0$ denotes $S C$ is safe. In this paper, we focus on three types of vulnerabilities:

Reentrancy is a well-known vulnerability that caused the infamous DAO attack. In Ethereum, when a smart contract function $F_{1}$ transfers money to a recipient contract $C_{1}$, the fallback function of $C_{1}$ will be automatically triggered. $C_{1}$ may invoke back to $F_{1}$ in its fallback function to reenter $F_{1}$ for stealing money. Since the current execution of $F_{1}$ waits for the transfer to finish, $C_{1}$ can make use of the intermediate state $F_{1}$ is in to succeed in stealing.

Infinite loop is a common vulnerability in smart contracts. The program of a function may contain an iteration or loop with no exit condition or the exit condition cannot be reached, i.e., an infinite loop. The fallback mechanism in smart contracts rises a new possibility of this non-termination bug, namely a cycled call between functions and the fallback function. For example, function $A$ invokes function $B$ with incorrect arguments, which will automatically trigger the execution of the fallback function in this contract. Suppose the fallback function further invokes function $A$, this will leads to a call loop between $A$ and the fallback function.

Timestamp dependence vulnerability exists when a smart contract uses the block timestamp as a triggering condition to execute some critical operations, e.g., sending Ether or de- termining the winner of a game. The miner in Ethereum has the freedom to set the timestamp of a block within a short time interval ( $<900$ seconds) [Jiang et al., 2018]. Therefore, miners may manipulate the block timestamps to gain illegal benefits.

\section{Our Method}

Method overview. The overall architecture of our method consists of three phases: (1) a graph generation phase, which extracts the control flow and data flow semantics from the source code and explicitly models the fallback mechanism, (2) a graph normalization phase inspired by $k$-partite graph, and (3) novel message propagation networks for vulnerability modeling and detection. Next, we introduce the three phases, respectively.

\subsection{Graph Generation}

Existing work [Allamanis et al., 2018] has shown that programs can be transformed into symbolic graph representations, which are able to preserve semantic relationships between program elements. Inspired by this, we formulate a smart contract function into a contract graph, and assign distinct roles to different program elements (nodes). Further, we construct edges by taking their temporal order into consideration. Figs. 1(a) \& (b) demonstrate a contract snippet and the graph constructed for its getBonus function, respectively.

Our first insight is that different program elements in a function are not of equal importance. Therefore, we extract three categories of nodes, i.e., major nodes, secondary nodes, and fallback nodes.

Major nodes construction. Major nodes symbolize the invocations to customized or built-in functions that are important for detecting the specific vulnerability. For example, for reentrancy vulnerability, a major node models the invocation to a transfer function or the built-in call.value function, which is key to detect reentrancy. For timestamp dependence vulnerability, the built-in function invocation block.timestamp is extracted as a major node. For infinite loop, all the customized functions within the contract are treated as major 


\begin{tabular}{|c|c|c|}
\hline Symbol & Semantic Fact & Type \\
\hline $\mathrm{AH}$ & $\operatorname{assert}\{X\}$ & \\
\hline RG & require $\{\mathrm{X}\}$ & \\
\hline IR & revert & \\
\hline IT & throw & \\
\hline IF & if $\{X\}$ & Control-flow edoes \\
\hline GB & if $\{\ldots\}$ else $\{X\}$ & \\
\hline GN & if $\{\ldots\}$ then $\{X\}$ & \\
\hline WH & while $\{X\} \operatorname{do}\{\ldots\}$ & \\
\hline FR & for $\{X\} \operatorname{do}\{\ldots\}$ & \\
\hline $\begin{array}{l}\text { AG } \\
\text { AC }\end{array}$ & $\operatorname{assign}\{X\}$ & Data-flow edges \\
\hline FW & $\frac{\operatorname{access}\{\Lambda\}}{\text { natural sequential relationships }}$ & Forward edge \\
\hline FB & interactions with fallback function & Fallback edge \\
\hline
\end{tabular}

Table 1: Semantic edges summarization. All edges are classified into 4 types, namely control-flow, data-flow, forward, and fallback.

nodes. Formally, we characterize all the critical functions as major nodes, which are denoted by $M_{1}, M_{2}, \ldots, M_{n}$.

Secondary nodes construction. While major nodes represent important invocations, secondary nodes are used to model critical variables, e.g., user balance and bonus flag. Formally, the critical variables are defined as secondary nodes $S_{1}, S_{2}, \ldots, S_{n}$.

Fallback node construction. Further, we construct a fallback node $F$ to stimulate the fallback function of an attack contract, which can interact with the function under test. The fallback function is a special design in smart contracts, and is the cause of many security vulnerabilities.

Edges construction. We further construct edges to model the relationships between nodes. Each edge describes a path that might be traversed through by the contract function under test, and the temporal number of the edge characterizes its order in the function. Specifically, the feature of an edge is extracted as a tuple $\left(V_{s}, V_{e}, o, t\right)$, where $V_{s}$ and $V_{e}$ represent its starting and end nodes, $o$ denotes its temporal order, and $t$ the edge type. To capture rich semantic dependencies between nodes, we construct four types of edges, namely control flow, data flow, forward and fallback edges. The details of the semantic edges are listed in Table 1.

\subsection{Contract Graph Normalization}

Most graph neural networks are inherently flat when propagating information, ignoring that some nodes play more central roles than others. Moreover, different contract source code yield distinct graphs, hindering the training of graph neural networks. Therefore, we propose a node elimination process to normalize graphs.

Nodes elimination. As introduced in Section 3.1, the node of a graph is partitioned into major nodes $\left\{M_{i}\right\}_{i=1}^{|M|}$, secondary nodes $\left\{S_{i}\right\}_{i=1}^{|M|}$, and the fallback node $F$. We remove each secondary node $S_{i}$ but pass the feature of $S_{i}$ to its nearest major node. Note that if $S_{i}$ has multiple nearest major nodes, its feature is passed to all of them. The fallback node is also removed similar to the secondary node. The edges connecting to the removed node are preserved but with their starting or end node moving to the corresponding major node.
Feature of major nodes. Features of major nodes are updated by aggregating features from their neighboring removed nodes. To distinguish between the original major node and its corresponding major node after aggregation, we denote the new major node of $M_{i}$ as $V_{i}$. The feature of $V_{i}$ is composed of three parts: i) self-feature, namely the feature of major node $M_{i}$; ii) in-features, namely features of the secondary nodes $\left\{P_{j}\right\}_{j=1}^{|P|}$ that are merged to $M_{i}$ and having a path pointing from $P_{j}$ to $M_{i}$; and iii) out-feature, namely features of the secondary nodes $\left\{Q_{k}\right\}_{k=1}^{|Q|}$ that are merged to $M_{i}$ and having a path directs from $Q_{k}$ from $M_{i}$. Fig. 1(c) illustrates the normalized graph of Fig. 1(b).

\subsection{Message Propagation Neural Networks}

In this subsection, we first extend the GCN to a degree-free GCN (DR-GCN), then propose a novel temporal message propagation network (TMP). Both the two proposed networks take the normalized graph $G$ of a smart contract function as input, and output the label $\hat{y} \in\{0,1\}$ indicating whether the function has a vulnerability of a certain type.

DR-GCN. [Kipf and Welling, 2017] proposes to apply convolutional neural networks to graph-structured data, which develops a layer-wise propagation network as:

$$
X_{l+1}=\sigma\left(\hat{D}^{-\frac{1}{2}} \hat{A} \hat{D}^{-\frac{1}{2}} X_{l} W_{l}\right)
$$

where $\hat{A}=A+I$ is the adjacency matrix $(A)$ enhanced with self-loops $(I), X_{l}$ is the feature matrix of layer $l$, and $W_{l}$ is a trainable weight matrix. In the equation, the diagonal node degree matrix $\hat{D}$ is used to normalize $\hat{A}$. We first increase the connectivity between nodes in the normalized graph $G$ by using the square of $A$. Then, we further take into account that the graph is already well normalized in our setting, and therefore remove matrix $\hat{D}$ from the equation. Finally, we arrive at the solution: $X_{l+1}=\sigma\left(\left(A^{2}+I\right) X_{l} W_{l}\right)$.

TMP. We also propose a TMP network, consisting of a message propagation phase and a readout phase (Fig. 2). In the message propagation phase, TMP passes information along the edges successively by following their temporal order. Then, TMP computes a label for the entire graph $G$ by using a readout function, which aggregates the final states of all nodes in $G$. Formally, $G=\{V, E\}$, where $V$ consists of all the major nodes and $E$ contains all the edges. Denote $E=$ $\left\{e_{1}, e_{2}, \ldots, e_{N}\right\}$, where $e_{k}$ represents the $k^{t h}$ temporal edge.

Message propagation phase. Messages are passed along the edges, one edge per time step. At time step 0, the hidden state $h_{i}^{0}$ for each node $V_{i}$ is initialized with the feature of $V_{i}$. At time step $k$, message flows through the $k^{t h}$ temporal edge $e_{k}$ and updates the hidden state of $V_{e k}$, namely the end node of $e_{k}$. Particularly, message $m_{k}$ is computed basing on $h_{s k}$, the hidden state of the starting node of $e_{k}$, and the edge type $t_{k}$ :

$$
\begin{array}{r}
x_{k}=h_{s k} \oplus t_{k} \\
m_{k}=W_{k} x_{k}+b_{k}
\end{array}
$$




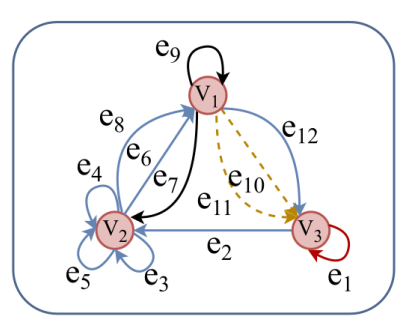

(a) Contract graph

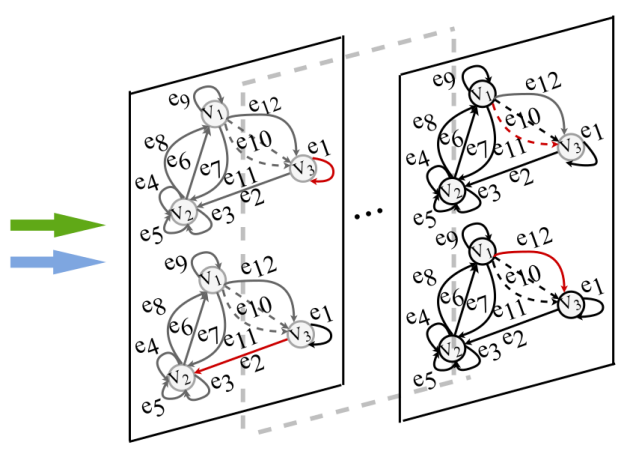

(b) Message propagation phase

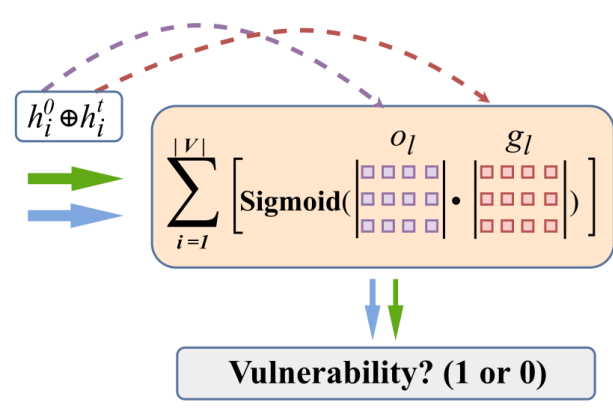

(c) Readout phase

Figure 2: The overall architecture of our proposed TMP. (a) The input normalized graph; b) The message propagation phase; (c) The readout phase that outputs the vulnerability detection result.

where $\oplus$ denotes concatenation operation, matrix $W_{k}$ and bias vector $b$ are network parameters. The original message $x_{k}$ contains information from the starting node of $e_{k}$ and edge $e_{k}$ itself, which are then transformed into a vector embedding using $W_{k}$ and $b$. After receiving the message, the end node of $e_{k}$ updates its hidden state $h_{e k}$ by aggregating information from the incoming message and its previous state. Formally, $h_{e k}$ is updated according to:

$$
\begin{array}{r}
\hat{h}_{e k}=\tanh \left(U m_{k}+Z h_{e k}+b_{1}\right) \\
h_{e k}^{\prime}=\operatorname{softmax}\left(R \hat{h}_{e k}+b_{2}\right)
\end{array}
$$

where $U, Z, R$ are matrices, while $b_{1}$ and $b_{2}$ are bias vectors. Readout phase. After successively traversing all the edges in $G$, TMP computes a label for $G$ by reading out the final hidden states of all nodes. Let $h_{i}^{T}$ be the final hidden state of the $i^{t h}$ node, we may generate the prediction label $\hat{y}$ by

$$
\hat{y}=\sum_{i=1}^{|V|} f\left(h_{i}^{T}\right)
$$

where $f$ is a mapping function, e.g., a neural network, and $|V|$ denotes the number of major nodes. However, we found that the differences between the final hidden state $h_{i}^{T}$ and the original hidden state $h_{i}^{0}$ are informative in the vulnerability detection task. Therefore, we instead consider to compute $\hat{y}$ as follows:

$$
\begin{aligned}
s_{i} & =h_{i}^{T} \oplus h_{i}^{0} \\
g_{i} & =\operatorname{softmax}\left(W_{g}^{(2)}\left(\tanh \left(b_{g}^{(1)}+W_{g}^{(1)} s_{i}\right)\right)+b_{g}^{(2)}\right) \\
o_{i} & =\operatorname{softmax}\left(W_{o}^{(2)}\left(\tanh \left(b_{o}^{(1)}+W_{o}^{(1)} s_{i}\right)\right)+b_{o}^{(2)}\right) \\
\hat{y} & =\sum_{i=1}^{|V|} \operatorname{Sigmoid}\left(o_{i} \odot g_{i}\right)
\end{aligned}
$$

where $\odot$ denotes element-wise product. $W_{j}, b_{j}^{(1)}$, and $b_{j}^{(2)}$, with subscript $j \in\{g, o\}$ are model parameters to be learned.

Both the two networks DR-GCN and TMP are trained for contract vulnerability detection. During training, networks are fed with a large number of normalized graphs constructed from smart contract functions, together with their ground truth labels. Then, the trained models are employed to absorb a normalized graph and yield a vulnerability detection label. We would like to point out that we developed automation tools for converting source code to normalized graphs, therefore, the whole procedure is fully automated.

\section{Experiments}

\subsection{Datasets and Experimental Settings}

Datasets. Extensive experiments are conducted on all the smart contracts that have source code on the Ethereum and VNT Chain platforms. We denote the two real-world smart contract datasets as ESC (Ethereum Smart Contracts) and VSC (VNT chain Smart Contracts), respectively.

- ESC consists of 40,932 Ethereum smart contracts with roughly 307,396 functions in total. Among the functions, around 5, 013 functions possess at least one invocation to call.value, making them potentially affected by the reentrancy vulnerability. Around 4, 833 functions contain the block.timestamp statement, making them susceptible to the timestamp dependence vulnerability.

- VSC consists of 4,170 smart contracts collected from the VNT Chain *, roughly containing 13, 761 functions. VNT Chain is an experimental public blockchain platform proposed by companies and universities from Singapore, China, and Australia.

Experimental settings. We compared our approaches (DR-GCN and TMP) with a total of twelve other methods, namely four existing smart-contract vulnerability detection methods (Oyente [Luu et al., 2016], Mythril [Mueller, 2017], Smartcheck [Tikhomirov et al., 2018], and Securify [Tsankov et al., 2018]), four neural network based methods (VanillaRNN, LSTM, GRU, and GCN), and four program loop detection methods (Jolt [Carbin et al., 2011], PDA [Ibing and Mai, 2015], SMT [Kling et al., 2012], and Looper [Burnim et al., 2009]). For each dataset, we randomly pick $20 \%$ contracts as the training set while the remainings are utilized for the testing set. In the comparison, metrics accuracy, recall, precision, and Fl score are all involved. In consideration of the

\footnotetext{
*Vntchain website, 2018. https://github.com/vntchain/go-vnt.
} 


\begin{tabular}{|c|c|c|c|c|c|c|c|c|c|c|c|c|c|}
\hline \multirow{2}{*}{ Methods } & \multicolumn{4}{|c|}{ Reentrancy } & \multicolumn{4}{|c|}{ Timestamp dependence } & \multirow{2}{*}{ Methods } & \multicolumn{4}{|c|}{ Infinite Loop } \\
\hline & $\overline{\operatorname{Acc}(\%)}$ & Recall(\%) & Precision $(\%)$ & $\mathrm{F} 1(\%)$ & $\operatorname{Acc}(\%)$ & Recall $(\%)$ & Precision $(\%)$ & $\mathrm{F} 1(\%)$ & & $\overline{\operatorname{Acc}(\%)}$ & Recall(\%) & Precision $(\%)$ & $\mathrm{F} 1(\%)$ \\
\hline Smartcheck & 52.97 & 32.08 & 25.00 & 28.10 & 44.32 & 37.25 & 39.16 & 38.18 & Jolt & 42.88 & 23.11 & 38.23 & 28.81 \\
\hline Oyente & 61.62 & 54.71 & 38.16 & 44.96 & 59.45 & 38.44 & 45.16 & 41.53 & PDA & 46.44 & 21.73 & 42.96 & 28.26 \\
\hline Mythril & 60.54 & 71.69 & 39.58 & 51.02 & 61.08 & 41.72 & 50.00 & 45.49 & SMT & 54.04 & 39.23 & 55.69 & 45.98 \\
\hline Securify & 71.89 & 56.60 & 50.85 & 53.57 & - & - & - & - & Looper & 59.56 & 47.21 & 62.72 & 53.87 \\
\hline Vanilla-RNN & 49.64 & 58.78 & 49.82 & 50.71 & 49.77 & 44.59 & 51.91 & 45.62 & Vanilla-RNN & 49.57 & 47.86 & 42.10 & 44.79 \\
\hline LSTM & 53.68 & 67.82 & 51.65 & 58.64 & 50.79 & 59.23 & 50.32 & 54.41 & LSTM & 51.28 & 57.26 & 44.07 & 49.80 \\
\hline GRU & 54.54 & 71.30 & 53.10 & 60.87 & 52.06 & 59.91 & 49.41 & 54.15 & GRU & 51.70 & 50.42 & 45.00 & 47.55 \\
\hline GCN & 77.85 & 78.79 & 70.02 & 74.15 & 74.21 & 75.97 & 68.35 & 71.96 & GCN & 64.01 & 63.04 & 59.96 & 61.46 \\
\hline DR-GCN & 81.47 & 80.89 & 72.36 & 76.39 & 78.68 & 78.91 & 71.29 & 74.91 & DR-GCN & 68.34 & 67.82 & 64.89 & 66.32 \\
\hline TMP & 84.48 & 82.63 & 74.06 & 78.11 & 83.45 & 83.82 & 75.05 & 79.19 & TMP & 74.61 & 74.32 & 73.89 & 74.10 \\
\hline
\end{tabular}

Table 2: Performance comparison in terms of accuracy, recall, precision, and F1 score. A total of fourteen methods are investigated in the comparison, including state-of-the-art vulnerability detection methods, neural network based alternatives, our methods DR-GCN and TMP. '-' denotes not applicable.

\begin{tabular}{|c|c|c|c|c|c|c|c|c|c|c|c|c|}
\hline \multirow{2}{*}{ Metrics } & \multicolumn{4}{|c|}{ Reentrancy } & \multicolumn{4}{|c|}{ Timestamp dependence } & \multicolumn{4}{|c|}{ Infinite loop } \\
\hline & $\overline{\text { DR-GCN-WON }}$ & DR-GCN & TMP-WON & TMP & DR-GCN-WON & DR-GCN & TMP-WON & TMP & DR-GCN-WON & DR-GCN & TMP-WON & TMP \\
\hline $\operatorname{Acc}(\%)$ & 77.08 & 81.47 & 81.91 & 84.48 & 72.56 & 78.68 & 80.03 & 83.45 & 63.93 & 68.34 & 70.03 & 74.61 \\
\hline Recall $(\%)$ & 76.83 & 80.89 & 80.49 & 82.63 & 74.38 & 78.91 & 81.30 & 83.82 & 63.16 & 67.82 & 71.82 & 74.32 \\
\hline Precision $(\%)$ & 68.36 & 72.36 & 71.44 & 74.06 & 67.46 & 71.29 & 72.69 & 75.05 & 60.11 & 64.89 & 69.94 & 73.89 \\
\hline $\mathrm{F} 1(\%)$ & 72.35 & 76.39 & 75.70 & 78.11 & 70.75 & 74.91 & 76.75 & 79.19 & 61.59 & 66.32 & 70.87 & 74.10 \\
\hline
\end{tabular}

Table 3: Accuracy comparison between DR-GCN, TMP, and their variants on the three vulnerability detection tasks.

distinct features of different platforms, experiments on reentrancy vulnerability and timestamp dependence vulnerability are conducted on the ESC dataset, while experiments on infinite loop vulnerability detection are conducted on the VSC dataset.

\subsection{Comparison with Existing Methods}

In this subsection, we first benchmark the proposed approaches (DR-GCN and TMP) against state-of-the-art methods on the reentrancy, timestamp dependence, and infinite loop vulnerabilities, respectively. Then, we compare our approaches with other neural network based methods.

\section{Comparison on Reentrancy Vulnerability Detection}

First, we compare our DR-GCN and TMP methods with state-of-the-art smart contract vulnerability detection methods, namely Oyente [Luu et al., 2016], Mythril [Mueller, 2017], Smartcheck [Tikhomirov et al., 2018], and Securify [Tsankov et al., 2018], on the reentrancy vulnerability detection task. The performance of different methods is presented in the left of Table 2, where metrics accuracy, recall, precision, and F1 score are engaged.

From the quantitative results of Table 2, we have the following observations. First, we find that existing tools have not yet achieved a satisfactory accuracy on reentrancy vulnerability detection, e.g., the state-of-the-art tool yields a $71.89 \%$ accuracy. Second, TMP outperforms state-of-the-art methods by a large margin. More specifically, TMP achieves an accuracy of $84.48 \%$, gaining a $12.39 \%$ accuracy improvement over state-of-the-art tools. Besides, the F1 score of TMP is $24.54 \%$ higher than existing methods. Thirdly, DR-GCN also achieves better results than other existing methods in terms of all the four metrics. The strong empirical evidences reveal the great potential of applying graph neural networks to smart contract vulnerability detection.

\section{Comparison on Timestamp Dependence Vulnerability Detection}

We then compare the proposed methods with state-of-the-art smart contract vulnerability detection tools on the timestamp dependence vulnerability detection task. The comparison results are demonstrated in the middle of Table 2 . The stateof-the-art method has obtained a $61.08 \%$ accuracy on timestamp dependence vulnerability detection, which is quite low. This may stem from the fact that most of existing methods detect timestamp dependence vulnerability by crudely checking whether there is block.timestamp statement in the function. Moreover, in consistent with the results on reentrancy vulnerability detection, TMP keeps delivering the best performance in terms of all the four metrics, while DR-GCN ranks the second. In particular, TMP gains a $22.37 \%$ accuracy improvement over state-of-the-art method.

We further look into the existing smart contract vulnerability detection tools to investigate the reasons behind the observations. Smartcheck fundamentally depends on a few rigid and simple logic rules to detect vulnerabilities, which leads to low accuracy and F1 score. Oyente employs data flow analysis to improve the accuracy, while its underlying patterns for detecting vulnerabilities are not so accurate. Regarding Mythril, it requires sophisticated techniques such as taint analysis or manual audit, which attains a medium accuracy. Unlike other methods, Securify classifies smart contract functions into violations, warnings, and compliances, where violation denotes the function is guaranteed to have the vulnerability (positive), and compliance denotes the function is safe (negative). We treat all warnings as negative since users are usually attracted by violations while ignoring a lot of warnings. Securify performs better than other existing methods, but has a high false negative rate.

\section{Comparison on Infinite Loop Vulnerability Detection}

For the infinite loop vulnerability detection, we compare our methods against available tools including Jolt [Carbin et al., 

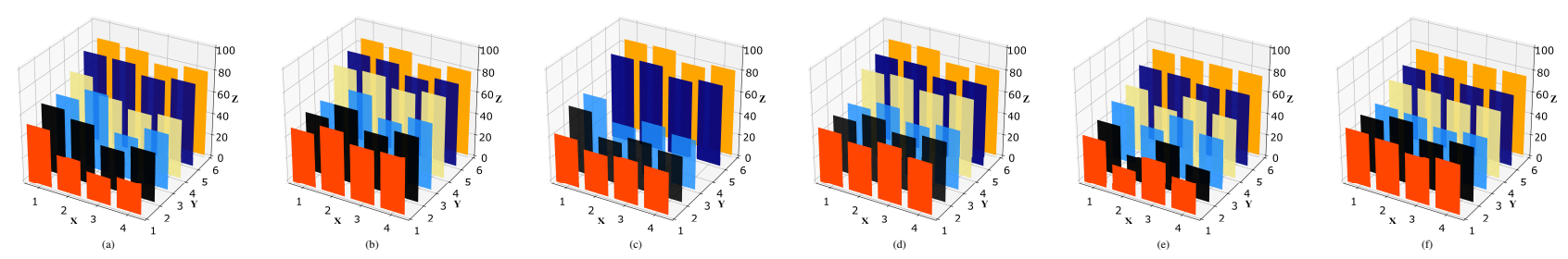

Figure 3: Visually comparison: (a) \& (b) present comparison results of reentrancy vulnerability detection on the ESC dataset, while (c) \& (d) present comparison results of timestamp dependence detection, (e) \& (f) show comparison results of infinite loop vulnerability detection on the VSC dataset. In (a) \& (c), the 6 rows from front to back denote the Smartcheck, Oyente, Mythril, Securify, DR-GCN, and TMP methods, respectively. In (e), the 5 rows from front to back denote the Jolt, PDA, SMT, Looper, DR-GCN, and TMP methods, respectively. In (b) \& (d) \& (f), the 6 rows from front to back denote the Vanilla-RNN, LSTM, GRU, GCN, DR-GCN, and TMP methods, respectively. For each row in the figures, accuracy, recall, precision, and F1 score are respectively demonstrated from left to right.

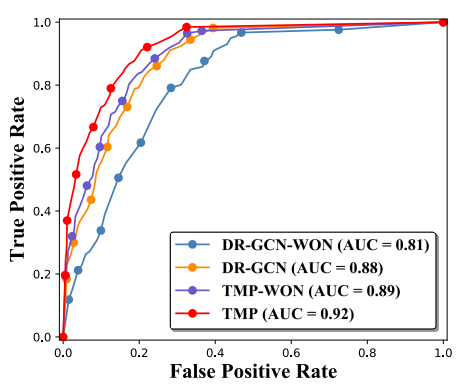

(a) Reentrancy

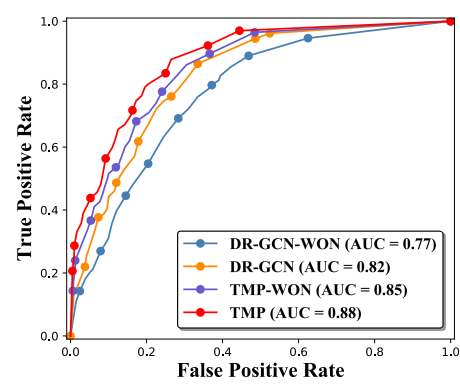

(b) Timestamp

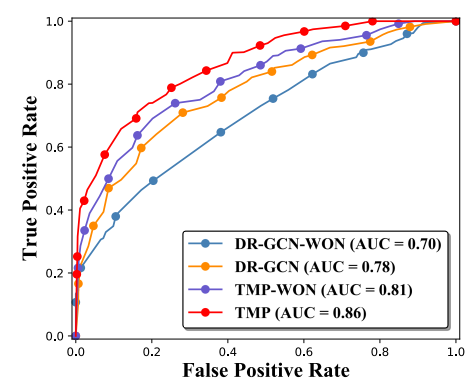

(c) Infinite loop

Figure 4: ROC analysis for DR-GCN, TMP, and their variants on the three vulnerability detection tasks. AUC stands for area under the curve.

2011], SMT [Kling et al., 2012], PDA [Ibing and Mai, 2015], and Looper [Burnim et al., 2009]. We empirically find that almost all existing methods fail to detect the infinite loop bug caused by the fallback mechanism of smart contracts. In contrast, our methods can successfully identify this vulnerability. This is because we explicitly model the fallback mechanism of smart contracts and consider data dependencies and control dependencies between program elements. Quantitative results are illustrated in the right of Table 2. From the table, we see that TMP consistently and significantly outperforms the other methods on the infinite loop vulnerability detection task. In particular, TMP and DR-GCN respectively achieve a $15.05 \%$ and $8.78 \%$ accuracy improvement over state-of-theart methods.

We further visualize the comparison results in Fig. 3(a), (c), and (e). Fig. 3(a) and Fig. 3(c) present comparison results of reentrancy vulnerability detection and timestamp dependence vulnerability detection, respectively. The 6 rows (in different colors) from front to back denote the Smartcheck, Oyente, Mythril, Securify, DR-GCN, and TMP methods, respectively. For each row in the figures, accuracy, recall, precision, and F1 score are respectively demonstrated from left to right. Fig. 3(e) shows comparison results of infinite loop vulnerability detection, where the the 6 rows from front to back denote the Jolt, PDA, SMT, Looper, DR-GCN, and TMP methods, respectively. We can clearly observe that DR-GCN and TMP outperform existing methods.

\section{Comparison with Neural Network Based Methods}

In order to seek out which neural network architectures could succeed in smart contract vulnerability detection, we also compare our methods with other neural network alternatives. Specifically, Vanilla-RNN, LSTM, GRU, and GCN are compared with our DR-GCN and TMP networks. For fair comparison, all the methods are presented with the vector representation of the normalized graph extracted from the source code and are required to detect the corresponding bugs. We illustrate the results of different models in terms of accuracy, recall, precision, and F1 score in Table 2. Fig. 3(b), (d), and (f) further visualize the results.

Interestingly, experimental results show that conventional recurrent neural networks Vanilla-RNN, LSTM, and GRU perform no better than existing vulnerability detection methods. In contrast, graph neural networks GCN, DR-GCN, and TMP, which are capable of handling graphs, achieve significantly better results than existing methods. This suggests that blindly treat the source code as a sequence is not suitable for the vulnerability detection task, while modeling the source code into graphs and adopting graph neural networks is promising. We conjecture that conventional recurrent models lose valuable information from smart contract code since they ignore the structural information of contract programs, such as the data-flow and invocation relationships.

We would like to highlight that the proposed TMP and $D R$ $G C N$ model consistently and significantly outperforms other neural network models in terms of all the 4 metrics. Besides TMP and DR-GCN, the GCN model performs the best. The 
accuracies of GCN and DR-GCN are lower than TMP. We attribute this to the fact that GCN fails to capture the temporal information induced by data flow and control flow, which is explicitly addressed in our TMP model using ordered edges.

\subsection{Study on The Effect of Graph Normalization}

By default TMP adopts the graph normalization module to highlight the major nodes in the graph, it is interesting to see the effect of removing this module. We removed the graph normalization phase from TMP and DR-GCN, and compared them with the default TMP and DR-GCN. The two variants are respectively denoted as TMP-WON and DR-GCN-WON, where WON is short for without normalization. Quantitative results are summarized in Table 3. We can see that with the proposed normalization module, the performance of both DR-GCN and TMP is better. For example, on the reentrancy vulnerability detection task, the DR-GCN model obtains a $4.39 \%$ and $4.04 \%$ improvement in terms of accuracy and F1 score, respectively, while TMP gains a $2.57 \%$ and $2.41 \%$ improvement in accuracy and F1 score.

Fig. 4 further plots the ROC curves of DR-GCN, TMP, and their variants. We adopt Receiver Operating Characteristic (ROC) analysis to show the impact of the graph normalization module. AUC (area under the curve) is used as the measure for performance, the higher AUC the better performance. Fig. 4(a) demonstrates that DR-GCN and TMP achieve better results on the reentrancy detection task with the normalization module, namely the AUC increases by 0.07 and 0.03 , respectively. Regarding the timestamp dependence detection task, DR-GCN and TMP obtain a 0.05 and 0.03 improvement in AUC (shown in Fig. 4(b)). For the infinite loop detection task, DR-GCN and TMP gain a 0.08 and 0.05 improvement in AUC (shown in Fig. 4(c)). The experimental results suggest that program elements should contribute distinctly in vulnerability detection rather than having equal contributions.

\section{Related Work}

Smart contract vulnerability detection. Smart contract vulnerability detection is one of the fundamental problems in blockchain security. Current work mainly relies on symbolic execution methods, such as Oyente [Luu et al., 2016], Maian [Nikolić et al., 2018] and Securify [Tsankov et al., 2018], which suffer from high false negative rates due to the inability to explore all possible program paths. Recent work [Jiang et $a l ., 2018$ ] explores dynamic execution for vulnerability detection, but requires a hand-crafted agent contract for reentrancy detection, preventing it from fully automated application.

Graph neural networks (GNNs). With remarkable success of neural networks in various fields [Cheng et al., 2019] [Liu et al., 2018a], graph neural network has received increasing attention. Existing approaches roughly cast into two categories: (1) Spectral-based approaches generalize wellestablished neural models like CNNs for graph data. For instance, GCN [Kipf and Welling, 2017] implements a firstorder approximation of spectral graph convolutions [Defferrard et al., 2016], while [Li et al., 2018] proposes a graph CNN capable of processing input data of arbitrary graph structure. (2) Spatial-based methods inherit ideas from recurrent GNNs and adopt message passing for graph convolutions. [Micheli, 2009] directly sums up a node's neighborhood information for graph convolutions, while recent work [Veličković et al., 2017] and [Zhang et al., 2018] learn different weights of neighboring nodes using attention mechanisms.

\section{Conclusion}

In this paper, we have proposed a fully automated vulnerability analyzer for smart contracts. In contrast to existing methods, we explicitly model the fallback mechanism of smart contracts, consider rich dependencies between program elements, and explore the possibility of using novel graph neural networks for vulnerability detection. Extensive experiments show that our method significantly outperforms state-of-theart methods and other neural networks. We believe our work is an important step towards revealing the potential of deep learning methods on smart contract vulnerability detection tasks.

\section{Acknowledgments}

This paper is supported by the National Key R\&D Program of China (2017YFB1401300, 2017YFB1401304), the National Natural Science Foundation of China (No. 61902348), the Natural Science Foundation of Zhejiang Province, China (Grant No. LQ19F020001).

\section{References}

[Allamanis et al., 2018] Miltiadis Allamanis, Marc Brockschmidt, and Mahmoud Khademi. Learning to represent programs with graphs. In International Conference on Learning Representations, 2018.

[Burnim et al., 2009] Jacob Burnim, Nicholas Jalbert, Christos Stergiou, and Koushik Sen. Looper: Lightweight detection of infinite loops at runtime. In Proceedings of the International Conference on Automated Software Engineering, pages 161-169. IEEE Computer Society, 2009.

[Carbin et al., 2011] Michael Carbin, Sasa Misailovic, Michael Kling, and Martin C Rinard. Detecting and escaping infinite loops with jolt. In European Conference on Object-Oriented Programming, pages 609-633. Springer, 2011.

[Cheng et al., 2019] Zhiyong Cheng, Xiaojun Chang, Lei Zhu, Rose Catherine Kanjirathinkal, and Mohan S. Kankanhalli. MMALFM: explainable recommendation by leveraging reviews and images. ACM Trans. Inf. Syst., 37(2):16:1-16:28, 2019.

[Defferrard et al., 2016] Michaël Defferrard, Xavier Bresson, and Pierre Vandergheynst. Convolutional neural networks on graphs with fast localized spectral filtering. In Advances in neural information processing systems, pages 3844-3852, 2016.

[Ibing and Mai, 2015] Andreas Ibing and Alexandra Mai. A fixed-point algorithm for automated static detection of infinite loops. In International Symposium on High Assurance Systems Engineering, pages 44-51. IEEE, 2015. 
[Jiang et al., 2018] Bo Jiang, Ye Liu, and WK Chan. Contractfuzzer: Fuzzing smart contracts for vulnerability detection. In Proceedings of the International Conference on Automated Software Engineering, pages 259-269. ACM, 2018.

[Kipf and Welling, 2017] Thomas N. Kipf and Max Welling. Semi-supervised classification with graph convolutional networks. In International Conference on Learning Representations, 2017.

[Kling et al., 2012] Michael Kling, Sasa Misailovic, Michael Carbin, and Martin Rinard. Bolt: on-demand infinite loop escape in unmodified binaries. ACM SIGPLAN Notices, 47(10):431-450, 2012.

[Li et al., 2018] Ruoyu Li, Sheng Wang, Feiyun Zhu, and Junzhou Huang. Adaptive graph convolutional neural networks. In Thirty-Second AAAI Conference on Artificial Intelligence, 2018.

[Liu et al., 2018a] An-An Liu, Ning Xu, Hanwang Zhang, Weizhi Nie, and Yuting Su. Multi-level policy and reward reinforcement learning for image captioning. 2018.

[Liu et al., 2018b] Chao Liu, Han Liu, Zhao Cao, Zhong Chen, Bangdao Chen, and Bill Roscoe. Reguard: finding reentrancy bugs in smart contracts. In Proceedings of International Conference on Software Engineering: Companion Proceeedings, pages 65-68. ACM, 2018.

[Luu et al., 2016] Loi Luu, Duc-Hiep Chu, Hrishi Olickel, Prateek Saxena, and Aquinas Hobor. Making smart contracts smarter. In Conference on computer and communications security, pages 254-269. ACM, 2016.

[Micheli, 2009] Alessio Micheli. Neural network for graphs: A contextual constructive approach. IEEE Transactions on Neural Networks, 20(3):498-511, 2009.

[Mueller, 2017] Bernhard Mueller. A framework for bug hunting on the ethereum blockchain. Webiste, 2017. https: //github.com/ConsenSys/mythril.

[Nikolić et al., 2018] Ivica Nikolić, Aashish Kolluri, Ilya Sergey, Prateek Saxena, and Aquinas Hobor. Finding the greedy, prodigal, and suicidal contracts at scale. In Proceedings of the Annual Computer Security Applications Conference, pages 653-663. ACM, 2018.

[Sankar et al., 2017] Lakshmi Siva Sankar, M Sindhu, and M Sethumadhavan. Survey of consensus protocols on blockchain applications. In International Conference on Advanced Computing and Communication Systems, pages 1-5. IEEE, 2017.

[Tikhomirov et al., 2018] Sergei Tikhomirov, Ekaterina Voskresenskaya, Ivan Ivanitskiy, Ramil Takhaviev, Evgeny Marchenko, and Yaroslav Alexandrov. Smartcheck: Static analysis of ethereum smart contracts. In International Workshop on Emerging Trends in Software Engineering for Blockchain, pages 9-16. IEEE, 2018.

[Tsankov et al., 2018] Petar Tsankov, Andrei Dan, Dana Drachsler-Cohen, Arthur Gervais, Florian Buenzli, and Martin Vechev. Securify: Practical security analysis of smart contracts. In Conference on Computer and Communications Security, pages 67-82. ACM, 2018.

[Veličković et al., 2017] Petar Veličković, Guillem Cucurull, Arantxa Casanova, Adriana Romero, Pietro Lio, and Yoshua Bengio. Graph attention networks. arXiv preprint arXiv:1710.10903, 2017.

[Zhang et al., 2018] Jiani Zhang, Xingjian Shi, Junyuan Xie, Hao Ma, Irwin King, and Dit-Yan Yeung. Gaan: Gated attention networks for learning on large and spatiotemporal graphs. arXiv preprint arXiv:1803.07294, 2018. 